\title{
Calculations of the Energetics of Oxidation of Aqueous Nucleosides and the Effects of Prototropic Equilibria
}

David M. Close*

Department of Physics, East Tennessee State Univ., Johnson City, TN 37614

Peter Wardman

formerly of the Gray Cancer Institute, CRUK/MRC Oxford Institute for Radiation Oncology, University of Oxford, Oxford OX3 7DQ, United Kingdom; present address: 20 Highover Park, Amersham, Buckinghamshire HP7 OBN, United Kingdom

\section{Supporting Information}

\section{1) Guanosine.}

Schroeder et al. ${ }^{1}$ computed standard reduction potential for guanosine from potentials derived at $\mathrm{pH} 7$ using equation (1).

$$
E^{0}\left(\mathrm{Nuc}^{+} / \mathrm{Nuc}\right)=E-\frac{R T}{F} \ln \left(\frac{1}{K_{\mathrm{a} 1}} \cdot \frac{\left[\mathrm{H}^{+}\right]^{3}+K_{\mathrm{a} 1}\left[\mathrm{H}^{+}\right]^{2}+K_{\mathrm{a} 1} K_{\mathrm{a} 2}\left[\mathrm{H}^{+}\right]+\mathrm{K}_{\mathrm{a} 1} K_{\mathrm{a} 2} K_{\mathrm{a} 3}}{\left[\mathrm{H}^{+}\right]^{2}+K_{\mathrm{r} 1}\left[\mathrm{H}^{+}\right]+\mathrm{K}_{\mathrm{r} 1} K_{\mathrm{r} 2}}\right)
$$

The $\mathrm{p} K$ as were taken from Steenken and Jovanovic and are $\mathrm{p} K_{\mathrm{r} 1}=1.9$ (involves deprotonation of the $\mathrm{N} 7+\mathrm{H}$ cation), $\mathrm{p} K_{\mathrm{r} 2}=9.25$ (involves deprotonation of the neutral guanosine), $\mathrm{p} K_{\mathrm{r} 3}=12.33$ (involves deprotonation of the $\mathrm{C}^{\prime}-\mathrm{OH}$ ), $\mathrm{p} K_{\mathrm{o} 1}=3.9$ (involves deprotonation of the guanosine radical cation), and $\mathrm{p} K_{\mathrm{o} 2}=10.9$ (involves deprotonation of $\mathrm{N} 1-\mathrm{H}$ to form an anion). Using these numbers yields the $E^{\mathrm{o}}=1.47 \mathrm{~V}$ as shown in Table 1 of the text.

The xyz coordinates for the Guanosine Neutral Radical:

Guanosine Neutral Radical Opt 6-31+G(d,p)

02

$\begin{array}{lrrr}8 & 3.216715 & -2.098739 & 0.324099 \\ 6 & 3.787911 & -1.465563 & -0.827346 \\ 1 & 3.545705 & -2.026782 & -1.738826 \\ 1 & 4.881010 & -1.397311 & -0.733156 \\ 6 & 3.221763 & -0.059905 & -0.955587 \\ 1 & 3.726364 & 0.443667 & -1.787629 \\ 8 & 1.809902 & -0.132454 & -1.265163 \\ 6 & 1.033093 & 0.493022 & -0.258494 \\ 1 & 0.660466 & 1.470032 & -0.584213\end{array}$




$\begin{array}{lrrr}7 & -0.142321 & -0.326040 & 0.000942 \\ 6 & -0.171435 & -1.675466 & 0.267059 \\ 1 & 0.746430 & -2.242524 & 0.349347 \\ 7 & -1.400677 & -2.175298 & 0.380400 \\ 6 & -2.225736 & -1.124068 & 0.167115 \\ 6 & -3.696379 & -1.008964 & 0.160126 \\ 8 & -4.437389 & -1.965932 & 0.383784 \\ 7 & -4.178698 & 0.262772 & -0.120053 \\ 6 & -3.352029 & 1.259767 & -0.342476 \\ 7 & -3.867024 & 2.486183 & -0.608906 \\ 1 & -4.866982 & 2.586690 & -0.685597 \\ 1 & -3.251925 & 3.248442 & -0.842621 \\ 7 & -1.947226 & 1.259287 & -0.334807 \\ 6 & -1.472665 & 0.065233 & -0.088559 \\ 6 & 3.345208 & 0.814477 & 0.300923 \\ 1 & 4.152173 & 0.476346 & 0.963034 \\ 6 & 1.969658 & 0.654492 & 0.962004 \\ 1 & 1.962465 & -0.261798 & 1.561931 \\ 8 & 1.694156 & 1.797014 & 1.754852 \\ 1 & 0.917495 & 1.644661 & 2.308088 \\ 8 & 3.562670 & 2.153955 & -0.118411 \\ 1 & 3.598648 & -2.979319 & 0.426891 \\ 1 & 3.275003 & 2.740663 & 0.597856\end{array}$

\section{2) Adenosine}

Schroeder et al. ${ }^{1}$ used equation (2) to calculate $\mathrm{E}^{\mathrm{o}}$ for adenosine using $\mathrm{p} K_{\mathrm{r} 1}=3.6$

(involves depronation of the $\mathrm{N} 7+\mathrm{H}$ cation), $\mathrm{p} K_{\mathrm{a} 2}=12.5$ (involves deprotonation of the neutral guanosine), and $\mathrm{p} K_{\mathrm{o} 1}=4.2$ (involves deprotonation of the guanosine radical cation at $\mathrm{N} 6$ to yield the N6-H radical).

$$
E^{0}\left(\mathrm{Nuc}^{++} / \mathrm{Nuc}\right)=E-\frac{R T}{F} \ln \left(\frac{1}{K_{\mathrm{a} 1}} \cdot \frac{\left[\mathrm{H}^{+}\right]^{2}+K_{\mathrm{a} 1}\left[\mathrm{H}^{+}\right]+\mathrm{K}_{\mathrm{a} 1} K_{\mathrm{a} 2}}{\left[\mathrm{H}^{+}\right]+\mathrm{K}_{\mathrm{r} 1}}\right)
$$

This yields the value $E^{0}=1.61$ listed in Table 1 of the text.

The xyz coordinates for the Adenosine Neutral Radical:

Adenosine N6-H Neutral Radical OPT 6-31+G(d,p)

02

$\begin{array}{llll}8 & 2.957219 & 1.561995 & -1.879430 \\ 6 & 3.722833 & 1.524494 & -0.681027 \\ 1 & 3.807570 & 2.524527 & -0.234141 \\ 1 & 4.727607 & 1.194882 & -0.962879 \\ 6 & 3.158821 & 0.559987 & 0.353062\end{array}$




$\begin{array}{lrrr}1 & 3.802628 & 0.554026 & 1.240569 \\ 8 & 1.837124 & 1.047955 & 0.740784 \\ 6 & 0.941414 & -0.041435 & 0.794347 \\ 1 & 0.959532 & -0.526619 & 1.780360 \\ 7 & -0.406048 & 0.489571 & 0.558358 \\ 6 & -0.846936 & 1.767680 & 0.828135 \\ 1 & -0.160883 & 2.525717 & 1.176589 \\ 7 & -2.138287 & 1.949690 & 0.609171 \\ 6 & -2.587375 & 0.728788 & 0.189654 \\ 6 & -3.885293 & 0.264631 & -0.200925 \\ 7 & -4.986065 & 1.007494 & -0.230569 \\ 1 & -4.739640 & 1.957292 & 0.072925 \\ 7 & -3.988716 & -1.061124 & -0.572691 \\ 6 & -2.917277 & -1.825021 & -0.555888 \\ 1 & -3.047493 & -2.861351 & -0.855663 \\ 7 & -1.638149 & -1.483314 & -0.206544 \\ 6 & -1.527646 & -0.210471 & 0.153112 \\ 6 & 2.962603 & -0.892869 & -0.118805 \\ 1 & 3.483027 & -1.089254 & -1.063741 \\ 6 & 1.438599 & -1.022213 & -0.288642 \\ 1 & 1.149054 & -0.634506 & -1.274653 \\ 8 & 1.050178 & -2.361217 & -0.110872 \\ 1 & 0.073992 & -2.398315 & -0.200467 \\ 8 & 3.431847 & -1.757898 & 0.906159 \\ 1 & 2.962107 & -2.601698 & 0.807329 \\ 1 & 2.113965 & 1.990983 & -1.681572\end{array}$

Viewing this file with a graphics utility one can see an intramolecular H-bond $\mathrm{C}_{2^{\prime}}-\mathrm{O}_{2^{\prime}}-\mathrm{H} \cdots \mathrm{N}_{3}=1.94 \AA$.

\section{3) Cytidine}

Likewise, Equation (3) was used to calculate the $E^{\mathrm{o}}$ for cytidine using $\mathrm{p} K_{\mathrm{a} 1}=4.2$ (involves deprotonation of the cytosine $\mathrm{N} 1+\mathrm{H}$ cation), and $\mathrm{p} K_{\mathrm{o} 1}=4.0$ (involves deprotonation of the $\mathrm{N} 1+\mathrm{H}$ radical cation .

$$
E^{0}\left(\mathrm{Nuc}^{-+} / \mathrm{Nuc}\right)=E-\frac{R T}{F} \ln \left(\frac{\left[\mathrm{H}^{+}\right] \cdot\left(K_{\mathrm{a} 1}+\left[\mathrm{H}^{+}\right]\right)}{K_{\mathrm{a} 1}\left(\left[\mathrm{H}^{+}\right]+\mathrm{K}_{\mathrm{r} 1}\right)}\right)
$$

This yields the value This yields the value $E^{0}=1.8$ listed in Table 1 of the text.

The xyz coordinates for the Cytidine Neutral Radical: 
Cytidine Neutral Radical OPT XYZ

02

$\begin{array}{lrrr}8 & 1.632701 & 2.158287 & -1.229461 \\ 6 & 2.556576 & 2.175861 & -0.139024 \\ 1 & 2.312631 & 2.982211 & 0.566387 \\ 1 & 3.587076 & 2.323496 & -0.492533 \\ 6 & 2.487638 & 0.844323 & 0.590095 \\ 1 & 3.216862 & 0.856685 & 1.406927 \\ 8 & 1.172754 & 0.675886 & 1.185283 \\ 6 & 0.507653 & -0.439421 & 0.650357 \\ 1 & 0.453005 & -1.256862 & 1.378784 \\ 7 & -0.900204 & -0.067966 & 0.350095 \\ 6 & -1.318142 & 1.221762 & 0.355525 \\ 1 & -0.552218 & 1.960583 & 0.546177 \\ 6 & -2.637976 & 1.514208 & 0.137986 \\ 1 & -3.008421 & 2.530020 & 0.129350 \\ 6 & -3.529950 & 0.410950 & -0.045900 \\ 7 & -4.852405 & 0.685965 & -0.195150 \\ 7 & -3.110103 & -0.865833 & -0.071193 \\ 6 & -1.784841 & -1.149122 & 0.048342 \\ 8 & -1.303418 & -2.282007 & -0.081173 \\ 6 & 2.728549 & -0.401737 & -0.272281 \\ 1 & 3.291527 & -0.164858 & -1.184318 \\ 6 & 1.307052 & -0.892291 & -0.602649 \\ 1 & 0.927719 & -0.358557 & -1.482683 \\ 8 & 1.327380 & -2.285721 & -0.798321 \\ 1 & 0.412215 & -2.603715 & -0.647951 \\ 8 & 3.420088 & -1.360603 & 0.509787 \\ 1 & 1.734811 & 2.963114 & -1.752157 \\ 1 & 3.099644 & -2.233245 & 0.223029 \\ 1 & -5.331261 & -0.217980 & -0.300903\end{array}$

Viewing this file with a graphics utility one can see an intramolecular H-bond $\mathrm{C}_{2^{\prime}}-\mathrm{O}_{2^{\prime}}-\mathrm{H} \cdots \mathrm{O}-\mathrm{C}_{2}=1.84 \AA$.

\section{4) Thymidine}

Finally, equation (4) was used to calculate $E^{0}=$ for thymidine using $\mathrm{p} K_{\mathrm{r} 1}=9.8$ (involves depronation of the neutral thymidine at $\mathrm{N} 1+\mathrm{H}$ ), and $\mathrm{p} K_{\mathrm{o} 1}=3.6$ (involves deprotonation of the radical cation at $\mathrm{N} 1-\mathrm{H})$. 


$$
E^{0}\left(\mathrm{Nuc}^{++} / \mathrm{Nuc}\right)=E-\frac{R T}{F} \ln \left(\frac{K_{\mathrm{a} 1}+\left[\mathrm{H}^{+}\right]}{\left[\mathrm{H}^{+}\right]+\mathrm{K}_{\mathrm{r} 1}}\right)
$$

This yields the value $E^{0}=1.9$ listed in Table 1 of the text.

The xyz coordinates for the Thymidine Neutral Radical:

Thymidine Neutral Radical Opt XYZ

02

$\begin{array}{lrrr}8 & -1.578874 & 2.265079 & 1.155333 \\ 6 & -2.497415 & 2.349766 & 0.062121 \\ 1 & -2.169276 & 3.107005 & -0.663018 \\ 1 & -3.505828 & 2.615005 & 0.409082 \\ 6 & -2.570836 & 0.999481 & -0.630713 \\ 1 & -3.278349 & 1.072892 & -1.463207 \\ 8 & -1.270658 & 0.667648 & -1.192210 \\ 6 & -0.763564 & -0.517121 & -0.637604 \\ 1 & -0.814573 & -1.351150 & -1.346435 \\ 7 & 0.668741 & -0.337631 & -0.326918 \\ 6 & 1.233060 & 0.862950 & -0.272295 \\ 1 & 0.582766 & 1.716691 & -0.427040 \\ 6 & 2.608915 & 0.975707 & -0.055770 \\ 6 & 3.414018 & -0.282781 & 0.003316 \\ 8 & 4.648029 & -0.172588 & 0.025644 \\ 7 & 2.773019 & -1.485235 & 0.055262 \\ 6 & 1.465718 & -1.591796 & -0.025537 \\ 8 & 0.760703 & -2.599922 & 0.101975 \\ 6 & -2.968169 & -0.188357 & 0.255031 \\ 1 & -3.512892 & 0.133729 & 1.152139 \\ 6 & -1.620403 & -0.833599 & 0.627248 \\ 1 & -1.186340 & -0.311339 & 1.489299 \\ 8 & -1.803756 & -2.200883 & 0.873144 \\ 1 & -0.935688 & -2.638855 & 0.733615 \\ 8 & -3.751099 & -1.078970 & -0.518961 \\ 1 & -3.561849 & -1.974693 & -0.190093 \\ 1 & -1.642567 & 3.064601 & 1.692229 \\ 6 & 3.278140 & 2.294113 & 0.093010 \\ 1 & 4.174235 & 2.330277 & -0.534831 \\ 1 & 2.609147 & 3.124803 & -0.146125 \\ 1 & 3.637358 & 2.418010 & 1.123744\end{array}$

Viewing this file with a graphics utility one can see an intramolecular H-bond 
$\mathrm{C}_{2^{\prime}}-\mathrm{O}_{2}-\mathrm{H} \cdots \mathrm{O}-\mathrm{C}_{2}=1.81 \AA$.

1. $\quad$ Schroeder, C. A.; Pluhařová, E.; Seidel, R.; Schroeder, W. P.; Faubel, M.; Slaviček, P.; Winter, B.; Jungwirth, P.; Bradforth, S. E., Oxidation Half-Reaction of Aqueous Nucleosides and Nucleotides via Photoelectron Spectroscopy Augmented by ab initio Calculations. J. Am. Chem. Soc. 2015, 137, 201-209. 